\title{
A Study on Applying Divergence-Free Condition in Solving the Volume Integral Equation with Loop Basis
}

\author{
Mao-Kun $\mathrm{Li}^{(1)}$ and Weng Cho Chew ${ }^{(1)}$ \\ (1) Center of Computational Electromagnetics and Electromagnetic Laboratory, \\ Department of Electrical and Computer Engineering, University of Illinois at Urbana- \\ Champaign, Urbana, IL 61801, http://www.ccem.uiuc.edu/
}

\section{Introduction}

Volume integral equation (VIE) solver using method of moments and Schaubert-WiltonGlisson (SWG) basis that was introduced by Schaubert et al [1] has been used in many applications to simulate the electromagnetic response of dielectric medium. Since the electric flux is inherently divergence-free, a lot of research has been done on incorporating this into the VIE solver to reduce the number of unknowns and improve the accuracy [2-5]. This paper will focus on using the solenoidal volume loop basis scheme. A search algorithm based on graph theory for the complete independent loop basis set will be reported. To improve the convergence, an expanded volume loop basis is proposed. This basis set is easier to construct and also improves the convergence of the iterative solvers.

\section{Formulations}

\section{Loop Basis from Volume Tetrahedral Mesh}

The volume loop basis was defined as [4]

$$
\mathbf{O}_{i}(\mathbf{r})=\sum_{k=1}^{N_{k}} \frac{\mathbf{L}_{k}}{V_{k}} f_{k}(\mathbf{r})
$$

where $N_{k}$ is the total number of tetrahedrons attached to the $i$ th edge and

$$
f_{k}(\mathbf{r})=\left\{\begin{array}{lc}
1, & \mathbf{r} \in T_{k} \\
0, & \text { elsewhere }
\end{array}\right.
$$

The vector $\mathbf{L}_{k}$ is parallel with the edge that is opposite to the common edge in the $k$ th tetrahedron. The divergence of this basis is 0 because the electric flux in each tetrahedron is a constant vector. An example of a loop basis function in three dimensions is shown in Fig. 1. At the boundary of the objects, a half loop basis can be defined associated with the boundary edges. It can also be considered as a full loop basis function with virtual outside tetrahedrons of relative permittivity $\varepsilon_{r}=1$. Given the tetrahedral mesh of a closed object, we can define loop basis functions along edges. The number of complete independent basis functions for a no-hole object obeys

$$
N_{\text {loop }}=N_{s}-N_{t}=N_{e}-N_{v}+1
$$

where $N_{S}$ is the number of faces, $N_{t}$ is the number of tetrahedrons, $N_{v}$ is the number of vertices, and $N_{e}$ is the number of edges. The basis set in Eq. (3) includes both the full basis and half basis functions.

A question to answer before applying the volume loop basis is on setting up the complete independent loop basis set from the geometrical mesh. The loop basis functions defined associated with every edge will not be independent of each other. Mendes and 
Kulkarni both noticed this problem. To address the problem, Mendes used Gram-Schmidt orthogonalization in [4]; Kulkarni applied Gaussian elimination in [3] to select the independent basis set. The computational complexity of these two methods is large, and the physics is hidden in the computation of matrices. Therefore, a more efficient, physical method is needed to construct the independent loop basis set.

There are at least two ways available to set up the minimum and complete basis set: basis reduction and generating tree. The basis reduction scheme is based on the fact that the new basis set after basis reduction is actually a set of loop basis. However, there are many overlaps between these loops that generate a poorly conditioned matrix. Although some orthogonalization scheme can be used to reduce the correlation among basis functions, the numerical complexity will increase as in [3,4]. The generating tree scheme is based on the connecting information in a geometrical mesh. It has been applied in searching for loop basis functions from surface mesh [6]. In this scheme, the geometrical mesh can be considered as an undirected graph (undigraph) with tetrahedrons as nodes and faces as edges. The outer space needs to be considered as one polyhedron, i.e., another node in the undigraph. A generating tree of this undigraph connects all the tetrahedrons with a minimal number of faces. The number of leftover faces is equal to the number of loop basis functions according to Eq. (3). Therefore, when every leftover face is put into the generating tree to construct a new undigraph, one loop basis function can be found from it. Similar to surface loop basis, the loop basis functions generated using this scheme may not be minimal, thus the algorithm of reducing the loop to a minimum is needed after that.

To reduce the complexity of minimizing the loop basis functions, another generating tree scheme can be used in volume mesh. Instead of constructing the graph of tetrahedrons and faces, nodes and edges in a geometrical mesh can also be used to set up an undigraph. The generating tree of this new undigraph links the $N_{v}$ nodes with $N_{v}-1$ edges. The number of leftover edges is just $N_{e}-N_{v}+1$. According to Eq. (3), this number is equal to the number of volume loop basis functions. Therefore, these leftover edges can form an independent volume loop basis set. The advantage of this scheme is that every loop basis function defined from it is a minimal one; we do not need the additional step of minimizing each loop basis function. Its limitation is that it is only valid for simply connected region, i.e., an object without holes. If the object is not simply connected, the second identity in Eq. (3) is not true.

Fig. 1 also shows one example of generating loop basis functions from a diamond shape geometry. The thin dot-dashed lines with arrows indicate a tree constructed by tetrahedrons and faces $T_{1} \rightarrow T_{2} \rightarrow T_{4} \rightarrow T_{3}$. The solid dots indicate the independent loop basis defined associated with the corresponding edges. The thick dashed lines indicate another generating tree linking nodes together through edges. It shows that the two trees can generate the same set of loop basis.

\section{Loop Basis on Every Edge}

Although loop basis functions keep the divergence free condition and the normal continuity of flux, the poorly conditioned matrix they generate is detrimental to iterative matrix solvers. Hence a remedy needs to be found. One solution to improving the convergence of iterative solvers is to expand this set to every possible candidate of loop basis functions. Obviously, the basis functions in this new set are not independent of each other any more. A null space exists in this matrix equation. However, this null space will not worsen the condition of the matrix solver but speed up the convergence of solutions when an iterative solver is used. Although it seems that introducing a null space to the matrix is harmful, it can be proved that this null space is not detrimental if only the right 
hand side vector is used as an initial guess. Beside a mathematical proof, the reason can also be analyzed heuristically: Expanded loop basis set gives more freedom for the solution of flux to converge to the correct result than the minimal basis set, hence iterative solvers converge faster. Beside the improvement on convergence, there are several advantages in using the expanded loop basis set. Because the loop basis functions are defined associated with every edge, the selection of a basis set is much simpler; there is no need to execute the particular algorithm to determine which loop to keep and which to abandon. In addition, the scheme to construct this expanded basis set is not restricted to simply connected objects, which makes it more general.

\section{Numerical Examples}

As shown in Fig. 2, the model in this simulation is a dielectric ring. $\varepsilon_{r}$ is set to be 4 in this example. This object is discretized into 3,331 tetrahedrons with 7,356 SWG basis functions (11.5 per wavelength in the medium) defined associated with every surface and 4,965 (10.05 per wavelength in the medium) loop basis functions are defined associated with every edge. The number of basis functions is reduced by $32.5 \%$. The incident $1 \mathrm{GHz}$ plane wave comes from $-\hat{\mathbf{z}}$ to $+\hat{\mathbf{z}}$ direction. The CGNR method is used in the matrix solver. It takes 159 iterations for the matrix equation of SWG basis to converge to $10^{-3}$ and 390 iterations for the equation of loop basis to converge to the same error criteria. In the results of RCS plotted in Fig. 2, the three curves are very close to each other. The solid line indicates the RCS computed using SWG basis with a denser mesh to represent the accurate solution. From this example, it validates our treatment for the object with holes when using expanded volume loop basis set associated every edge. Therefore, unlike surface loop basis functions, volume basis functions could be defined on every edge directly without executing the complicated search algorithms. Furthermore, the construction of the basis functions is straightforward and faster than that of the surface loop basis.

\section{References:}

[1] D. Schaubert, D. Wilton, and A. Glisson, "A Tetrahedral Modeling Method for Electromagnetic Scattering by Arbitrarily Shaped Inhomogeneous Dielectric Bodies," IEEE Trans Antenn Propag, vol. 32, pp. 77-85, 1984.

[2] H.-Y. Chao, "A Multilevel Fast Multipole Algorithm for Analyzing Radiation and Scattering from Wire Antennas in a Complex Environment," University of Illinois at Urbana-Champaign, 2002.

[3] S. Kulkarni, R. Lemdiasov, R. Ludwig, and S. Makarov, "Comparison of Two Sets of Low-order Basis Functions for Tetrahedral VIE Modeling," IEEE Trans Antenn Propag, vol. 52, pp. 2789-2794, 2004.

[4] L. Mendes and S. Carvalho, "Scattering of EM Waves by Homogeneous Dielectrics with the Use of the Method of Moments and 3D Solenoidal Basis Functions," Microw Opt Tech Lett, vol. 12, pp. 327-331, 1996.

[5] B. Rubin, "Divergence-Free Basis For Representing Polarization Current in Finite-Size Dielectric Regions," IEEE Trans Antenn Propag, vol. 41, pp. 269277, 1993.

[6] W. C. Chew, Fast and Efficient Algorithms in Computational Electromagnetics. Boston: Artech House, 2001. 

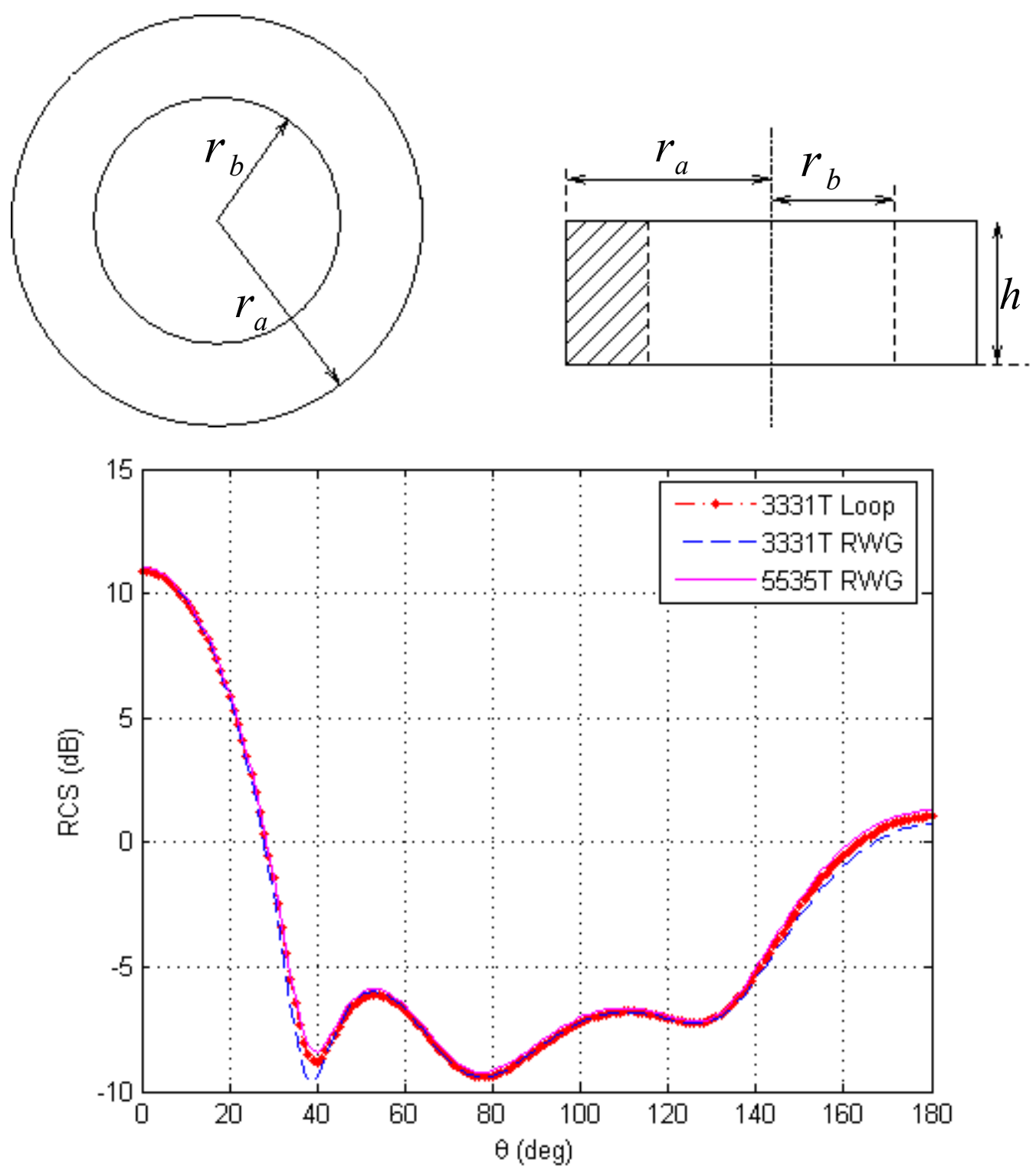

Figure 1 RCS of a dielectric ring with plane wave incident, $r_{a}=0.25 \mathbf{m}, r_{b}=h=0.1 \mathbf{m}$ 